\title{
UFO: a web server for ultra-fast functional profiling of whole genome protein sequences Peter Meinicke
}

Address: Department of Bioinformatics, Institute of Microbiology and Genetics, Georg-August-University Göttingen, Germany Email: Peter Meinicke - pmeinic@gwdg.de

Published: 2 September 2009

BMC Genomics 2009, 10:409 doi:10.1 |86/147|-2164-10-409
Received: 27 April 2009

Accepted: 2 September 2009

This article is available from: http://www.biomedcentral.com/147I-2/64/10/409

(c) 2009 Meinicke; licensee BioMed Central Ltd.

This is an Open Access article distributed under the terms of the Creative Commons Attribution License (http://creativecommons.org/licenses/by/2.0), which permits unrestricted use, distribution, and reproduction in any medium, provided the original work is properly cited.

\begin{abstract}
Background: Functional profiling is a key technique to characterize and compare the functional potential of entire genomes. The estimation of profiles according to an assignment of sequences to functional categories is a computationally expensive task because it requires the comparison of all protein sequences from a genome with a usually large database of annotated sequences or sequence families.

Description: Based on machine learning techniques for Pfam domain detection, the UFO web server for ultra-fast functional profiling allows researchers to process large protein sequence collections instantaneously. Besides the frequencies of Pfam and GO categories, the user also obtains the sequence specific assignments to Pfam domain families. In addition, a comparison with existing genomes provides dissimilarity scores with respect to 821 reference proteomes. Considering the underlying UFO domain detection, the results on 206 test genomes indicate a high sensitivity of the approach. In comparison with current state-of-the-art HMMs, the runtime measurements show a considerable speed up in the range of four orders of magnitude. For an average size prokaryotic genome, the computation of a functional profile together with its comparison typically requires about 10 seconds of processing time.

Conclusion: For the first time the UFO web server makes it possible to get a quick overview on the functional inventory of newly sequenced organisms. The genome scale comparison with a large number of precomputed profiles allows a first guess about functionally related organisms. The service is freely available and does not require user registration or specification of a valid email address.
\end{abstract}

\section{Background}

The assignment of genes to certain functional categories is a central task in genome annotation. The distribution of assignments, i.e. the functional profile, provides a highly informative summary of a genome. Functional profiling plays a key role in comparative genomics for studying aspects of systems biology on a genome wide scale [1]. Without the restriction of DNA sequencing to culturable organisms, metagenomics allows to study the genomic potential of whole microbial communities. Functional profiling of metagenomes is an essential tool for comparative analysis of microbial ecosystems [2]. In the context of functional genomics, gene clusters and protein domains are widely used for homology-based annotation. Both approaches cover different aspects of the annotation and are often used in parallel to obtain a comprehensive 
description. While gene clusters as used for COGs [3] or within the SEED framework [4] provide a valuable resource for functional annotation based on the identification of homologous genes, the domain based approach is focussed on modelling and detection of functional modules which usually involve only parts of a gene. At the level of functional modules, the Pfam domain family database [5] currently provides the highest coverage. State-of-the-art methods for protein domain detection, like HMMER [6], are computationally expensive and several approximation techniques have been suggested to accelerate the model based prediction of protein domains. With a slight loss of sensitivity fast prefiltering methods can achieve speed ups of about two orders of magnitude as compared with HMMER [7]. Computational speed is of particular importance for the design of web-based sequence analysis tools. Due to computational expense most web servers for protein domain search only provide a single-sequence submission interface $[8,9]$. In addition to single sequence submission, the Pfam web server [5] also offers a batch option which allows the user to submit small multiple fasta files. These files are restricted to a maximum of 1000 protein sequences with a maximum sequence length of 2000 residues.

Using machine learning techniques for feature-based protein sequence classification [10-12], the UFO web server for ultra-fast functional profiling provides an instantaneous estimation of Pfam profiles, i.e. frequencies of Pfam domains, for large sets of protein sequences. With a speed up of four orders of magnitude, UFO is well prepared to cope with the rapidly growing amount of genomic and metagenomic sequence data.

\section{Construction and content}

The UFO web server has been built around an efficient implementation of machine learning techniques for protein sequence classification which have been described in [10-12]. Fast feature-based techniques for protein sequence representation have been combined with a multi-class multi-label approach [12] to assign protein sequences to Pfam domain families. While our previous model was obtained from training with about $1.5 \times 10^{5}$ sequences from the Pfam A release 22 seed alignments, UFO is based on training with the complete Pfam A release 23 full alignments which comprise more than $6 \times$ $10^{6}$ domain sequences. As an important difference, our previous publication [12] only considers a prefiltering method that uses the family specific scores from feature space discriminants to produce a ranking of domain models which in turn can be used to reduce the set of HMMER models in subsequent searches. UFO also uses a highdimensional word-based feature space [11] according to a word length of 20 amino acids, but in addition the discri- minant scores of the five highest scoring domain families are passed to a small neural network to decide whether a score actually indicates a valid match. The neural network architecture and its training has been described in [13] for the case of metagenomic gene prediction. UFO uses a network with five hidden units and with three inputs which correspond to the particular discriminant score and the mean and maximum score over all models. The output corresponds to an estimated posterior probability of a true match. Currently, domain families with a probability above 0.5 are reported as valid matches. In comparison with profile hidden Markov models [6], the feature-based machine learning approach does not provide a localization of protein domains but merely an indication of the presence or absence of a certain domain within a protein sequence. This implies that also the order or the repetition of domains cannot be predicted by the utilized approach. However, for the purpose of functional profiling this kind of "pure" domain detection usually does not mean a limitation. Actually, it has been shown that the prediction of protein function can be realized fairly well without considering domain repetitions or the ordering of domains [14]. For reasons of speed, another restriction as compared with Pfam/HMMER arises from the maximum number of domain families which can be detected within a single sequence. Currently, a protein sequence can be assigned to at most five different families. Only in rare cases we observed that this number was exceeded in the existing annotations.

In the first instance, the UFO server provides an ultra-fast search engine for detection of protein domains [5] according to the Pfam A release 23 from July 2008 which comprises 10340 domain families. In addition, UFO contains the precomputed profiles of 821 genomes from the HAMAP database (release from March 2009) [15] which are used for profile comparison. These reference genomes include 54 archaeal, 721 bacterial, and 46 eukaryotic proteomes, respectively. The complete list of reference genomes can be found in one of the UFO output files which are described in the next section. As a dissimilarity measure UFO utilizes the "profile divergence" with respect to these proteomes, which is computed in terms of Jeffreys' J-divergence, a symmetrized version of the Kullback-Leibler divergence between two probability distributions [16]. Given two profiles $P, Q$ with estimated domain probabilities $p_{i}, q_{i}$ for $n$ domain families the profile divergence is

$$
D(P, Q)=\sum_{i=1}^{n}\left(p_{i} \log \frac{p_{i}}{q_{i}}+q_{i} \log \frac{q_{i}}{p_{i}}\right)
$$

The probabilities are estimated from the corresponding domain frequencies using a pseudocount parameter $c$. A 
suitable value of $c$ was determined by hierarchical cluster analysis based on the above divergence measure. For that purpose, a complete linkage clustering was applied to a collection of 1017 prokaryotic profiles from 21 different phyla. To cope with the typical database bias towards particular culturable organisms [17], from all profiles that correspond to the same genus only the medoid profile, which by definition yields the minimal sum of divergences to the members of that genus, was selected for clustering. For a varying pseudocount parameter with 101 logarithmically spaced values in the interval $\left[10^{-8}, 10^{2}\right]$ and each partition in the range between 10 and 50 clusters the agreement of the clustering with the given taxonomic groups on phylum level was measured by the adjusted Rand index [18]. The best agreement was obtained for a pseudo count $c=0.01$ with 22 clusters which resulted in a maximal adjusted Rand index of 5.17. For that partition the maximal within cluster divergence was $d_{c}=3.53$. This value is actually used by the UFO server to scale the profile divergence by $D(P, Q) / d_{c}$ to a more meaningful range, where values clearly below 1 usually correspond to phylogenetically and functionally related organisms.

\section{Utility and discussion User interface}

Considering the functionality of the UFO web server application, its use proceeds in the following manner: first, the user submits a collection of protein sequences in multiple fasta format either by pasting into the sequence input window or by uploading a valid multiple fasta file (see Figure 1). The maximum overall input size is 30 Mbyte. Sequences longer than 5000 amino acids are truncated and a corresponding warning is displayed. After submission the status of the processing is displayed in intervals of two seconds until the output files have been written. Then the results are shown on a new page which

\section{UFO [job submission]}

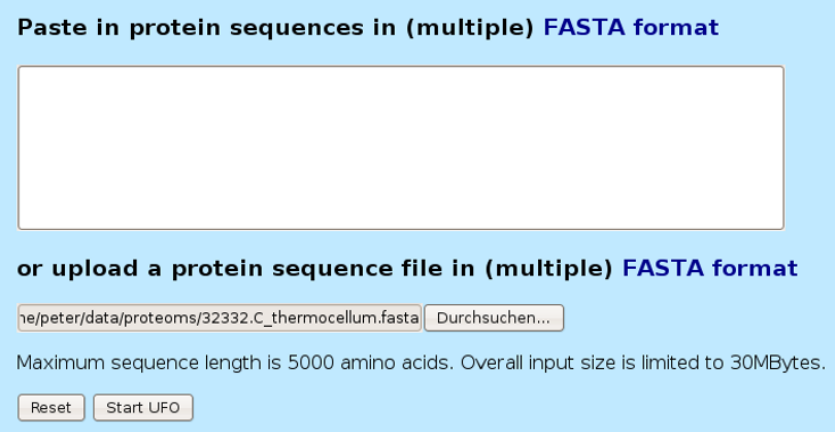

\section{Figure I}

Screenshot: UFO job submission. Screenshot of the UFO job submission page. provides several statistics, links for additional information, and a download section (see Figure 2). The statistics comprise runtime, the total number of detected domains and a top ten list of the most abundant domain families together with hyperlinks to the corresponding Pfam family description. Further information about the functional profile can be obtained on two additional results pages. The first page shows a more detailed view on the UFO assignments (see Figure 3): in a scrollable window, for each input sequence (fasta header) the Pfam domain assignments are displayed together with the associated Gene Ontology [19] annotation. While the name of the Pfam and GO categories is shown directly, the corresponding identifiers provide hyperlinks to a detailed description of the associated categories. In addition, the output probability score of the neural network is shown for the particular assignment. The value is in the range between 0.5 and 1.0 with high values above 0.9 usually indicating good matches. On another page the 10 nearest species in terms of the profile divergence with respect to a collection of precomputed reference profiles are shown in a sorted list (see Figure 4). The genome identifiers in that list provide hyperlinks to the corresponding HAMAP description for further information about the associated species. The complete list of 821 reference species together with the corresponding profile divergences (in ascending order) can be obtained as a text file by some link at the bottom of that page.

In the "Downloads" section of the main results page, several output files are available in plain ascii format. In addition a Perl script "ufo2hmmer" for postprocessing of the UFO assignments by means of selected HMMER/Pfam searches can be obtained. This script requires local HMMER and Perl installations and can be used to further increase the specificity of the domain detection. In addition, postprocessing of UFO matches with HMMER provides additional information about sequence positions, repetitions, and the order of the domains.

\section{Output files}

The first output file contains the complete Pfam profile in terms of domain specific detection counts sorted in descending order. The second file contains the corresponding GO profile which shows the assignment frequencies with respect to Gene Ontology categories. The GO counts result from applying the Pfam2GO mapping to the Pfam profile and again the frequencies are shown in descending order. The third file contains the sequence specific assignments to Pfam domain families together with the corresponding GO annotation and the match probability score of the neural network. This file may also be used for further processing, e.g. for Pfam/HMMER search of the UFO detected domains using the provided "ufo2hmmer" script. 


\section{UFO [Results UFO-1840896447]}

Main results page $\quad$ Assignments $\quad$ Nearest species $\quad$ New submission

| Maximum allowed sequence length is 5000 residues - oversized input has been truncated!

Processing of 2916 sequences took 7.2 seconds

Found 3021 assignments to Pfam domains in 2087 sequences

\section{Top 10 Pfams}

- PF00404 «Dockerin type I repeat» found in $\mathbf{6 7}$ sequences.

- PF00005 «ABC transporter» found in $\mathbf{6 1}$ sequences.

- PF00072 «Response regulator receiver domain» found in $\mathbf{3 8}$ sequences.

- PF04055 «Radical SAM superfamily» found in $\mathbf{3 6}$ sequences.

- PF07833 «Copper amine oxidase N-terminal domain» found in $\mathbf{3 6}$ sequences.

- PF02518 «Histidine kinase-, DNA gyrase B-, and HSP90-like ATPase» found in $\mathbf{3 4}$ sequences.

- PF00037 «4Fe-4S binding domain» found in $\mathbf{2 3}$ sequences.

- PF00528 «Binding-protein-dependent transport system inner membrane component» found in 18 sequences.

- PF00942 «Cellulose binding domain» found in $\mathbf{1 8}$ sequences.

- PF04542 «Sigma-70 region 2» found in 18 sequences.

View sequence specific assignments and nearest species from a set of 821 reference genomes.

\section{Downloads}

Get text files with functional profiles according to Pfam and GO counts, the corresponding list of sequence specific assignments and a Perl script for selective postprocessing with HMMER on your local computer:

- Pfam Profile

- GO Profile

- Assignments

- ufo $2 \mathrm{hmmer}$

submit another job

Figure 2

Screenshot: UFO example results. Screenshot of an UFO example "main results" page based on $C$. thermocellum (strain DSM 4I50) proteome.

\section{Performance analysis}

The performance of UFO was evaluated in two aspects: first, the accuracy of profiling was measured in terms of the underlying domain detection sensitivity and specificity on whole genome protein collections. Secondly, the speed of UFO was measured in terms of the run time required for the profiling of proteomes. To avoid direct overlap with the training sequences from the Pfam 23 (July 2008) release, I used the (multiple fasta) protein sequence files of 206 genomes from the "latest species" section of the Integr8 web site [20] where these genomes have been included since release 90/91 from January/February 2009. For a complete list of the test genomes see Additional file 1. Sensitivity and specificity were measured by means of a comparison with the available InterPro [21] Pfam hits from the Integr8 $\mathrm{ftp}$ site. Considering a single protein sequence, a true positive (TP) is counted if a protein domain family that has been detected by UFO is among the InterPro reference hits. A false negative (FN) occurs if the domain of a reference hit is overseen by UFO and a false positive (FP) is counted if an UFO detected domain family does not occur in the corresponding InterPro reference. For each of the 206 genomes the sensitivity is estimated by \#TP/(\#TP+\#FN) and the specificity by \#TP/(\#TP+\#FP). The mean (median) sensitivity and specificity over all genomes is 97.7 (98.8) and 81.1 (81.8), respectively. The histograms in Figure 5 show that by far in most cases the sensitivity is above 95\% with a slightly worse distribution of the specificity. However, because of the high sensitivity, UFO is well suitable for prefiltering. By selective postprocessing with a Pfam/HMMER search for the UFO detected domains (see "ufo2hmmer" in download section) a $100 \%$ specificity according to the above definition can easily be achieved. With an average number of 3750 genes, UFO profiling of all 206 genomes took about half an hour corresponding to an average runt- 


\title{
UFO [Assignments UFO-1840896447]
}

\author{
Main results page $\quad$ Assignments $\quad$ Nearest species $\quad$ New submission
}

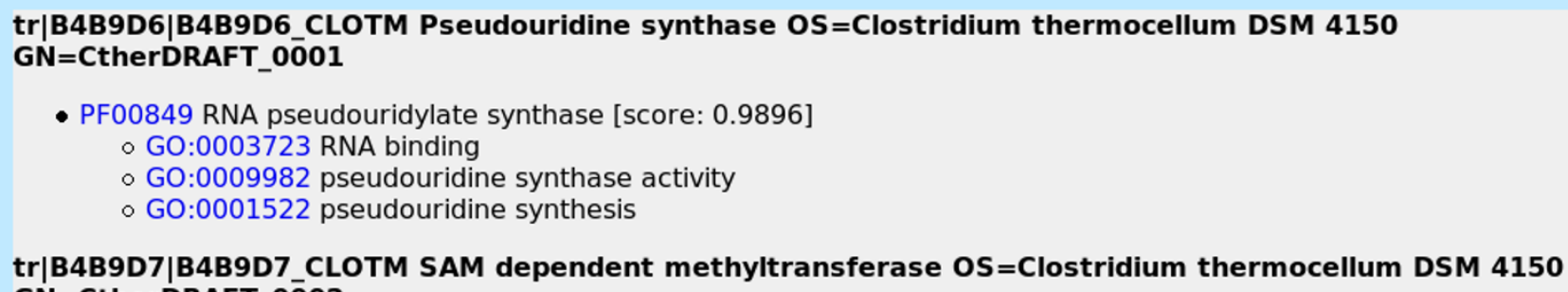

- PF00849 RNA pseudouridylate synthase [score: 0.9896]

- GO:0003723 RNA binding

- GO:0009982 pseudouridine synthase activity

- GO:0001522 pseudouridine synthesis

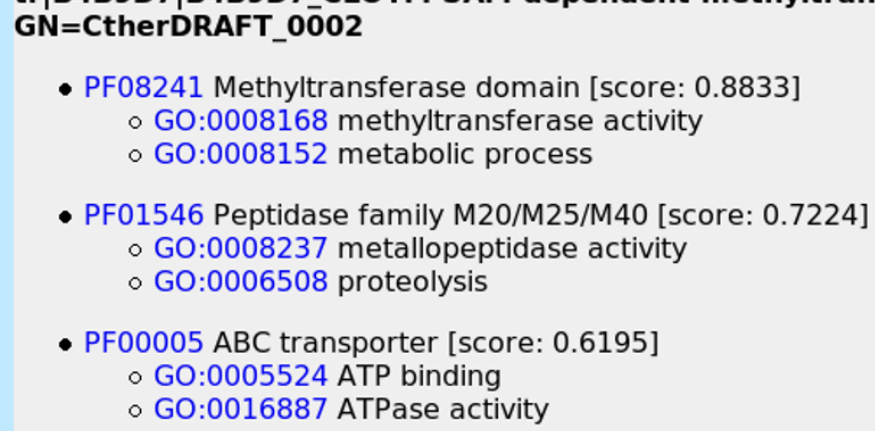

- PF08241 Methyltransferase domain [score: 0.8833]

- GO:0008168 methyltransferase activity

- GO:0008152 metabolic process

- PF01546 Peptidase family M20/M25/M40 [score: 0.7224]

- GO:0008237 metallopeptidase activity

- GO:0006508 proteolysis

- PF00005 ABC transporter [score: 0.6195]

- GO:0005524 ATP binding

- GO:0016887 ATPase activity

\section{tr|B4B9D8|B4B9D8_CLOTM Carbohydrate binding family 25 OS=Clostridium thermocellum DSM 4150 GN=CtherDRAFT_0003}

- PF03423 Carbohydrate binding domain (family 25)) [score: 0.9895]

o ohydrate binding domain (family 25)

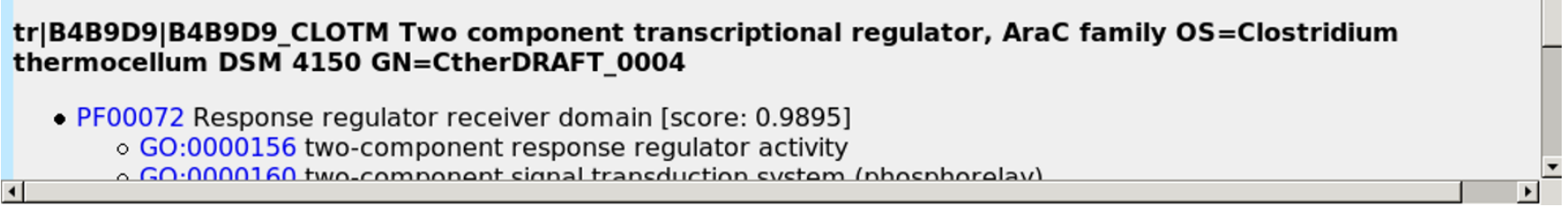

\section{Figure 3}

Screenshot: UFO assignments. Screenshot of an UFO example "assignments" page based on C. thermocellum (strain DSM 4I50) proteome.

ime of 9.7 seconds per genome. For comparison the RPSBLAST tool with the conserved domains database [9], which is widely used for accelerated protein domain searches, was locally installed and applied to the 206 test proteomes. All Pfam 23 domain searches were performed with default parameters and an E-value threshold of 0.001. Comparing the results with the InterPro reference, RPS-BLAST showed a mean (median) sensitivity of 90.6 (90.7) percent and a mean (median) specificity of 69.8 (70.2) percent, which are significantly lower than the corresponding UFO rates. The histograms in Figure 6 show a low variation of the sensitivity but for the specificity some outliers at the lower end increase the range of values. Referring to the runtime, RPS-BLAST took 59 CPU hours for processing of all 206 proteomes, with an average runt- ime of 17 minutes. Thus, UFO is about 100 times faster than RPS-BLAST.

Table 1 shows a comparison of runtimes for five of the smallest genomes which could also be processed by the batch option at the Pfam web site. The HMMER (version 2.3.2, Oct 2003), RPS-BLAST (version 2.2.16, Mar 2007), and UFO runtimes were measured as (single thread) user times on the same CPU (AMD Opteron 2.0 GHz) showing an UFO speed up of about four and two orders of magnitude if compared to HMMER and RPS-BLAST, respectively. This speed up makes it possible to pass UFO results directly to the user, whereas the Pfam web server (March 2009) with an average processing time of more than 8 hours for the small genomes sends the results by email. 


\title{
UFO [Nearest Species UFO-1840896447]
}

\author{
Main results page $\quad$ Assignments $\quad$ Nearest species $\quad$ New submission
}

The nearest species from a reference set of 821 genomes have been derived by means of a profile divergence which measures the dissimilarity between profiles by Jeffreys J-divergence. The J-divergence corresponds to a symmetrized version of the Kullback-Leibler divergence between two probability distributions. A suitable scaling of the profile divergence provides better interpretability, such that values clearly below 1 are likely to indicate a functionally related organism.

\section{Top 10 Nearest Species}

- cLOTH «Clostridium thermocellum» with profile divergence $\mathbf{0 . 0 9 7 7}$

- cals8 «Caldicellulosiruptor saccharolyticus» with profile divergence $\mathbf{0 . 5 5 9 2}$

- CLOAB «Clostridium acetobutylicum» with profile divergence $\mathbf{0 . 5 8 4 0}$

- CLOK5 «Clostridium kluyveri» with profile divergence $\mathbf{0 . 6 2 5 3}$

- cLOPH «Clostridium phytofermentans» with profile divergence $\mathbf{0 . 6 5 1 2}$

- THETN «Thermoanaerobacter tengcongensis» with profile divergence $\mathbf{0 . 6 7 7 3}$

- THEPX «Thermoanaerobacter sp» with profile divergence $\mathbf{0 . 6 8 0 0}$

- ALKMO «Alkaliphilus metalliredigens» with profile divergence $\mathbf{0 . 6 8 1 7}$

- cLONN «Clostridium novyi» with profile divergence $\mathbf{0 . 6 8 8 9}$

- HELMI «Heliobacterium modesticaldum» with profile divergence $\mathbf{0 . 7 0 5 1}$

Download file with a complete list of all divergences.

submit another job

Figure 4

Screenshot: UFO nearest species. Screenshot of an UFO example "nearest species" page based on $C$. thermocellum (strain DSM 4I50) proteome.

\section{Example application and discussion}

For an example application the proteome of a novel Clostridium thermocellum strain (DSM 4150, Integr8 ID: 32332) from the above collection of 206 test genomes was used to demonstrate the servers capabilities. Specifying the multiple fasta file of protein sequences on the UFO job submission page (see Figure 1) and pressing the "Start UFO" button initiates uploading and subsequent analysis of that file. After the processing of all 2916 sequences
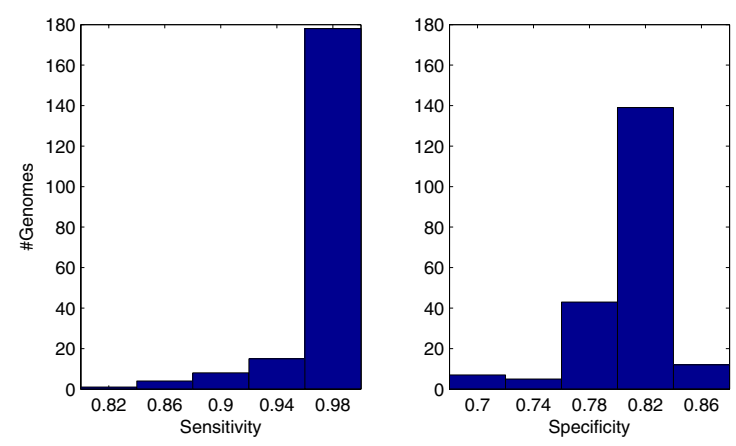

Figure 5

UFO domain detection performance. Histograms of UFO protein domain detection sensitivity and specificity. which takes about 7 seconds, the results page is generated and displayed. The results are based on 3021 assignments to Pfam domains which have been found in a total number of 2087 sequences. This implies that no domains have been found for 829 sequences. Besides the "assignments" page (see Figure 3) and the output files (hyperlinks) which allow a more detailed analysis of the profile properties, the "top ten" lists provide a brief summary of
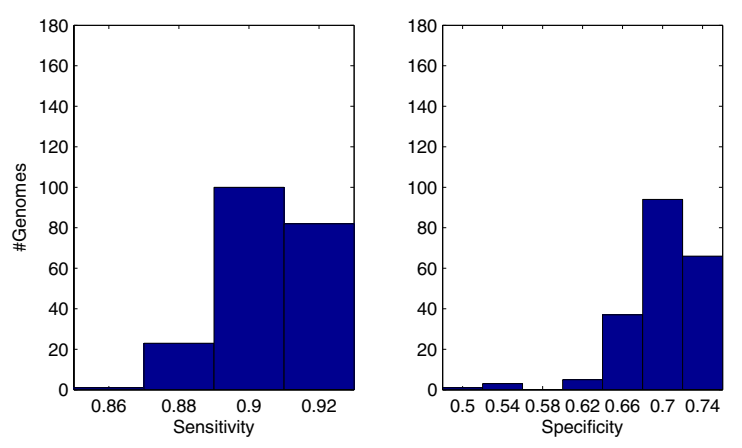

Figure 6

RPS-BLAST domain detection performance. Histograms of RPS-BLAST protein domain detection sensitivity and specificity. 
Table I: Runtime comparison for five small genomes between HMMER, RPS-BLAST, UFO and batch search at the Pfam web site (March 2009).

\begin{tabular}{|c|c|c|c|c|c|}
\hline \multirow[b]{2}{*}{ Species } & \multirow[b]{2}{*}{ \# Proteins } & \multicolumn{4}{|c|}{ CPU time } \\
\hline & & HMMER & RPS-BLAST & Pfam-web & UFO \\
\hline A. pseudotrichon. & 847 & 19 h 37 m & $4 \mathrm{~m} \mathrm{II} \mathrm{s}$ & $9 \mathrm{~h} 28 \mathrm{~m}$ & $2.5 \mathrm{~s}$ \\
\hline E. chaffeensis & 803 & 17 h 58 m & $3 \mathrm{~m} \mathrm{l} 3 \mathrm{~s}$ & $9 \mathrm{~h} 19 \mathrm{~m}$ & $2.0 \mathrm{~s}$ \\
\hline U. parvum & 577 & 13 h 54 m & $3 \mathrm{~m} 46 \mathrm{~s}$ & $7 \mathrm{~h} 38 \mathrm{~m}$ & $1.8 \mathrm{~s}$ \\
\hline U. urealyticum & 611 & $14 \mathrm{~h} 36 \mathrm{~m}$ & $3 \mathrm{~m} 42 \mathrm{~s}$ & $8 \mathrm{~h} 03 \mathrm{~m}$ & $1.9 \mathrm{~s}$ \\
\hline W. endosymbiont & 746 & 16 h 49 m & $3 \mathrm{~m} 40 \mathrm{~s}$ & $8 \mathrm{~h} 42 \mathrm{~m}$ & $1.9 \mathrm{~s}$ \\
\hline
\end{tabular}

the most prevalent features. In the example (see Figure 2), the most abundant Pfam family is the "Dockerin type I repeat" PF00404 which has been found in 67 sequences. Clicking the identifier shows the corresponding Pfam description of the family which indicates a key role of that domain in cellulose metabolization. Among the top 10 Pfams, also the "Cellulose binding domain" PF00942 found in 18 sequences indicates the importance of cellulose metabolism. The first entry of the nearest species list (see Figure 4) corresponds to another strain of C. thermocellum (ATCC 27405/DSM 1237) with a slightly bigger proteome set including 3102 proteins. According to the corresponding HAMAP description (hyperlink), C. thermocellum is a gram-positive, anaerobic, and thermophilic organism capable of cellulose degradation. The remaining species in the list also belong to the class of Clostridia, most of them are thermophilic. The closest five species are all able to ferment organic substrates.

As indicated by the application example above, the strength of UFO is its capability to produce a quick overview on the functional inventory of whole genomes in terms of the most abundant protein domains and in terms of the closest organisms with the most similar profiles. In comparison to full annotation servers like RAST [22] the UFO server only covers a particular aspect of genome annotation. It merely provides a first step of a functional analysis which can nevertheless be of great utility for addressing many biological questions and problems. It is not restricted to the analysis of prokaryotic genomes, and it can be applied to eukaryotes as well, if gene predictions are available. The runtimes for complete eukaryotic proteomes are usually above the average runtime for prokaryotes. For example, the proteome set of D. melanogaster (15410 sequences) takes 64 seconds of processing time, the C. elegans proteome ( 22984 sequences) requires 80 seconds. In addition, UFO can also be used to annotate large collections of (translated) expressed sequence tags. For prokaryotes the UFO domain detection can be used as a basis for the prediction of operons or regulons. Furthermore, the server supports researchers in the identification of functionally related species that can be used for annotation. For the analysis of microbial communities, gene prediction tools specialized on short anonymous DNA fragments $[13,23,24]$ or a simple six-frame translation can be used to apply UFO in functional metagenomics. In comparison to the more comprehensive MG-RAST server [25], UFO provides an easy-to-use interface with immediate response. For example, UFO profiling of the first of ten depth-specific data sets from the hypersaline microbial mat metagenome [26], which contains 12218 sequencing reads with an average length of $700 \mathrm{bp}$, requires $75 \mathrm{sec}-$ onds for processing of the six-frame translated reads. The processing of all ten data sets takes about 15 minutes. Inspection of the top ten Pfams shows a remarkable count for sulfatase (PF00884) assignments in lower layers with a maximum of 135 assignments in the fifth layer (4-5 mm depth), which is in accordance with the results in [26]. In general, the profile divergence with respect to the reference genomes is of limited use for metagenome analysis because a metagenomic profile actually corresponds to a mixture of several different species. However, if the habitat is dominated by a few closely related species, the UFO "top 10 nearest species" list may nevertheless be informative. In case of the hypersaline microbial mat the UFO results indicate a dominant role of Cyanobacteria in the two upmost layers $(0-1 \mathrm{~mm}$ and $1-2 \mathrm{~mm})$ with 9 and 6 out of 10 nearest species, respectively. This observation is in agreement with the analysis in [26] which indicates that Cyanobacteria together with Alphaproteobacteria are the most abundant phyla in these layers. Especially in metagenomics, the GO profile may facilitate the analysis because Pfam assignments are accumulated in categories, which directly relate to the biological questions. For example, considering the frequencies of the "chemotaxis" term (GO:0006935) for the hypersaline microbial mat data, the maximum count ( 55 assignments) is found in the third layer $(2-3 \mathrm{~mm})$ at the oxic-anoxic boundary, which agrees well with the original study [26].

\section{Conclusion}

With a considerable speed up of protein domain detection, UFO shows a new perspective in web-based large scale analysis of protein sequence data. As a consequence of its speed it can be used for instantaneous profiling of genome scale protein sequence files. The processing time 
roughly corresponds to the duration of a single sequence analysis as provided by current protein database servers. On the scale of prokaryotic genomes, UFO can process thousands of whole genome protein sets a day. In that way UFO is well prepared for next generation sequencing technologies like single cell sequencing [27], which allows to extract whole genomes from highly diverse metagenomic samples.

\section{Availability and requirements}

The UFO web service is freely available at http:// ufo.gobics.de.

\section{Authors' contributions}

PM designed the database interface and the search engine and wrote the manuscript. The author read and approved the final manuscript.

\section{Additional material}

\section{Additional file 1}

Test genomes used for performance evaluation. The file shows a list of all 206 test genomes that were used for evaluation of the UFO web server. The complete proteomes were obtained from recent Integr 8 data base updates and correspond to all entries of the "latest species" section which were added in release 90/91 from January/February 2009.

Click here for file

[http://www.biomedcentral.com/content/supplementary/14712164-10-409-S1.pdf]

\section{Acknowledgements}

I would like to thank Thomas Lingner for fruitful discussions and lots of technical support, Rasmus Steinkamp for web server and cluster support, Katharina Hoff for proof reading and Fabian Schreiber for Perl scripting.

\section{References}

I. Galperin M, Kolker E: New metrics for comparative genomics. Curr Opin Biotechnol 2006, I 7(5):440-447.

2. Dinsdale EA, Edwards RA, Hall D, Angly F, Breitbart M, Brulc JM, Furlan M, Desnues C, Haynes M, Li L, McDaniel L, Moran MA, Nelson KE, Nilsson C, Olson R, Paul J, Brito BR, Ruan Y, Swan BK, Stevens R, Valentine DL, Thurber RV, Wegley L, White BA, Rohwer F: Functional metagenomic profiling of nine biomes. Nature 2008 , 452:629-632.

3. Tatusov RL, Natale DA, Garkavtsev IV, Tatusova TA, Shankavaram UT, Rao BS, Kiryutin B, Galperin MY, Fedorova ND, Koonin EV: The COG database: new developments in phylogenetic classification of proteins from complete genomes. Nucleic Acids Res 200I, 29:22-28.

4. Overbeek R, Begley T, Butler RM, Choudhuri JV, Chuang HY, Cohoon M, de Crecy-Lagard V, Diaz N, Disz T, Edwards R, Fonstein M, Frank ED, Gerdes S, Glass EM, Goesmann A, Hanson A, IwataReuyl D, Jensen R, Jamshidi N, Krause L, Kubal M, Larsen N, Linke B, McHardy AC, Meyer F, Neuweger H, Olsen G, Olson R, Osterman A, Portnoy V, Pusch GD, Rodionov DA, Ruckert C, Steiner J, Stevens R, Thiele I, Vassieva O, Ye Y, Zagnitko O, Vonstein V: The subsystems approach to genome annotation and its use in the project to annotate $\mathbf{1 0 0 0}$ genomes. Nucleic Acids Res 2005, 33(I 7):5691-702.
5. Finn R, Tate J, Mistry J, Coggill P, Sammut J, Hotz H, Ceric G, Forslund K, Eddy S, Sonnhammer E, Bateman A: The Pfam protein family database. Nucleic Acids Res 2008, 36(suppl_I):D28I-D288.

6. Eddy S: Profile hidden Markov models. Bioinformatics 1998 I4(9):755-763.

7. Portugaly E, Ninio M: HMMERHEAD - Accelerating HMM Searches On Large Databases. Proc. Eighth Ann. Int'I Conf. Computational Molecular Biology (RECOMB) - Poster Abstracts 2004:250-25I.

8. Quevillon E, Silventoinen V, Pillai S, Harte N, Mulder N, Apweiler R, Lopez R: InterProScan: protein domains identifier. Nucleic Acids Res 2005, 33(suppl_2):WI I6-I20.

9. Marchler-Bauer A, Panchenko AR, Shoemaker BA, Thiessen PA, Geer LY, Bryant SH: CDD: a database of conserved domain alignments with links to domain three-dimensional structure. Nucleic Acids Res 2002, 30:28I-283.

10. Lingner T, Meinicke P: Remote homology detection based on oligomer distances. Bioinformatics 2006, 22( I 8):2224-3I.

II. Lingner T, Meinicke P: Word correlation matrices for protein sequence analysis and remote homology detection. BMC Bioinformatics 2008, 9:259.

12. Lingner T, Meinicke P: Fast Target Set Reduction for LargeScale Protein Function Prediction: A Multi-class Multi-label Machine Learning Approach. Algorithms in Bioinformatics, 8th International Workshop, WABI, Lecture Notes in Computer Science 2008, 525 I : 198-209.

13. Hoff KJ, Tech M, Lingner T, Daniel R, Morgenstern B, Meinicke P: Gene prediction in metagenomic fragments: a large scale machine learning approach. BMC Bioinformatics 2008, 9:217.

14. Forslund K, Sonnhammer E: Predicting protein function from domain content. Bioinformatics 2008, 24(1 5): I68I-I687.

15. Lima T, Auchincloss AH, Coudert E, Keller G, Michoud K, Rivoire C Bulliard V, de Castro E, Lachaize C, Baratin D, Phan I, Bougueleret L, Bairoch A: HAMAP: a database of completely sequenced microbial proteome sets and manually curated microbial protein families in UniProtKB/Swiss-Prot. Nucleic Acids Res 2009, 37(suppl_I):D47I-8.

16. Jeffreys $\mathrm{H}$ : Theory of Probability third edition. Oxford: Clarendon Press; 1961 .

17. Hugenholtz P: Exploring prokaryotic diversity in the genomic era. Genome Biology 2002, 3(2):reviews0003. I-reviews0003.8.

18. Hubert L, Arabie P: Comparing partitions. Journal of Classification 1985, 2:193-218.

19. Ashburner M, Ball CA, Blake JA, Botstein D, Butler H, Cherry JM, Davis AP, Dolinski K, Dwight SS, Eppig JT, Harris MA, Hill DP, IsselTarver L, Kasarskis A, Lewis S, Matese JC, Richardson JE, Ringwald M, Rubin GM, Sherlock G: Gene ontology: tool for the unification of biology. The Gene Ontology Consortium. Nature Genet 2000, 25:25-29.

20. Kersey P, Bower L, Morris L, Horne A, Petryszak R, Kanz C, Kanapin A, Das U, Michoud K, Phan I, Gattiker A, Kulikova T, Faruque N, Duggan K, Mclaren P, Reimholz B, Duret L, Penel S, Reuter I, Apweiler R: Integr8 and Genome Reviews: integrated views of complete genomes and proteomes. Nucleic Acids Res 2005, 33(suppl_I):D297-302.

21. Mulder NJ, Apweiler R, Attwood TK, Bairoch A, Bateman A, Binns D, Bork P, Buillard V, Cerutti L, Copley R, Courcelle E, Das U, Daugherty L, Dibley M, Finn R, Fleischmann W, Gough J, Haft D, Hulo N, Hunter S, Kahn D, Kanapin A, Kejariwal A, Labarga A, LangendijkGenevaux PS, Lonsdale D, Lopez R, Letunic I, Madera M, Maslen J, McAnulla C, McDowall J, Mistry J, Mitchell A, Nikolskaya AN, Orchard S, Orengo C, Petryszak R, Selengut JD, Sigrist CJA, Thomas PD, Valentin F, Wilson D, Wu CH, Yeats C: New developments in the InterPro database. Nucleic Acids Res 2007, 35(suppl_I):D224-8.

22. Aziz RK, Bartels D, Best AA, Dejongh M, Disz T, Edwards RA Formsma K, Gerdes S, Glass EM, Kubal M, Meyer F, Olsen G], Olson R, Osterman AL, Overbeek RA, McNeil LK, Paarmann D, Paczian T, Parrello B, Pusch GD, Reich C, Stevens R, Vassieva O, Vonstein V, Wilke A, Zagnitko O: The RAST Server: rapid annotations using subsystems technology. BMC Genomics 2008, 9:75.

23. Besemer J, Borodovsky M: Heuristic approach to deriving models for gene finding. Nucleic Acids Res 1999, 27( I 9):391 I-3920.

24. Noguchi H, Park J, Takagi T: MetaGene: prokaryotic gene finding from environmental shotgun sequences. Nucleic Acids Res 2006 , 34( I 9):5623-5630. 
25. Meyer F, Paarmann D, D'Souza M, Olson R, Glass E, Kubal M, Paczian T, Rodriguez A, Stevens R, Wilke A, Wilkening J, Edwards R: The metagenomics RAST server - a public resource for the automatic phylogenetic and functional analysis of metagenomes. BMC Bioinformatics 2008, 9:386.

26. Kunin $V$, Raes J, Harris J, Spear J, Walker J, Ivanova N, von Mering $C$, Bebout B, Pace N, Bork P, Hugenholtz P: Millimeter-scale genetic gradients and community level molecular convergence in a hypersaline microbial mat. Mol Syst Biol 2008, 4:198.

27. Woyke T, Xie G, Copeland A, González JM, Han C, Kiss H, Saw JH, Senin P, Yang C, Chatterji S, Cheng JF, Eisen JA, Sieracki ME, Stepanauskas $\mathrm{R}$ : Assembling the marine metagenome, one cell at a time. PLoS ONE 2009, 4:e5299.

Publish with Bio Med Central and every scientist can read your work free of charge

"BioMed Central will be the most significant development for disseminating the results of biomedical research in our lifetime. "

Sir Paul Nurse, Cancer Research UK

Your research papers will be:

- available free of charge to the entire biomedical community

- peer reviewed and published immediately upon acceptance

- cited in PubMed and archived on PubMed Central

- yours - you keep the copyright

Submit your manuscript here:

http://www.biomedcentral.com/info/publishing_adv.asp 